32. The problem of classifying three-dimensional manifolds.

33. Particular three-dimensional manifolds. Riemann spaces. Poincaré spaces.

Virgil SNyder.

\title{
NOTE ON THE ORDER OF CONTINUITY OF FUNCTIONS OF LINES.
}

BY DR. CHARLES ALBERT FISCHER.

(Read before the American Mathematical Society, Septernber 4, 1916.)

IT has been proved that if a linear function of a line has continuity of the zeroth order, it can be expressed as the limit of a sequence of definite integrals, ${ }^{*}$ as an integral of Stieltjes, $\dagger$ or as a Lebesgue integral. $\ddagger$ As has been remarked by Bliss, $\$$ many of the functions occurring in the calculus of variations do not have such continuity. The object of the present note is to show that if a function $U[y(x)]$ is linear and has continuity of the $n$th order, and if $y(x)$ is of class $C^{(n)}$, then $U[y(x)]$ is equal to the sum of a linear function of $d^{n} y(x) / d x^{n}$ which has continuity of the zeroth order, and a function of the values of $y(x)$ and its derivatives at an end point of the curve considered.

The proof is very simple. If $y(x)$ is of class $C^{(n)}$ it can be expressed as

$$
\begin{aligned}
y(x)=\int_{a}^{x} d x_{1} \int_{a}^{a x_{1}} d x_{2} \cdots \int_{a_{s}}^{x_{n-1}} y^{(n)}\left(x_{n}\right) d x_{n} \\
\quad+\sum_{i=1}^{n} \frac{y^{(n-i)}(a)}{(n-i) !}(x-a)^{n-i} .
\end{aligned}
$$

Then, since $U[y(x)]$, is linear,

$$
U\left[\begin{array}{c}
\stackrel{b}{y}(x) \\
a
\end{array}\right]=V\left[y^{(n)} \stackrel{b}{\underset{a}{a})}\right]+\sum_{i=1}^{n} \frac{y^{(n-i)}(a)}{(n-i) !} U\left[(x-\underset{a}{a})^{n-i}\right],
$$

where the function

$$
V\left[y^{(n)}(x)\right]=U\left[\int_{a}^{x} d x_{1} \cdots \int_{a}^{x_{n-1}} y^{(n)}\left(x_{n}\right) d x_{n}\right]
$$

* Hadamard, "Leçons sur le Calcul des Variations," p. 299.

$\dagger$ Riesz, Annales Scientifiques de L'Ecole Normale Supérieure, vol. 28 (1911), p. 43.

$\ddagger$ Fréchet, Transactions Amer. Math. Society, vol. 15 (1914), p. 140.

$\S$ Bliss, Proc. Nat. Acad. Sciences, vol. 1 (1915), p. 173. 
is seen to be linear and continuous of the zeroth order, and $U\left[(x-a)^{n-i}\right]$ is a constant.

To illustrate this, the function $U[y(x)]$ will be taken as the first variation of the integral

$$
J=\int_{a}^{b} f\left(x, y, y^{\prime}\right) d x,
$$

when $y(x)$ is replaced by $y(x)+\epsilon \eta(x)$. That is,

$$
U[\eta]=\epsilon \int_{a}^{b}\left(f_{y} \eta+f_{y^{\prime}} \eta^{\prime}\right) d x .
$$

If $f\left(x, y, y^{\prime}\right)$ and $y(x)$ are of class $C^{(2)}$, and $\eta(a)=\eta(b)=0$, this may be written

$$
U[\eta]=\epsilon \int_{a}^{b}\left(f_{y}-\frac{d}{d x} f_{y^{\prime}}\right) \eta d x
$$

If $f\left(x, y, y^{\prime}\right)$ and $y(x)$ are only of class $C^{\prime}$, this equation is not valid. In this case

$$
U[\eta]=V\left[\eta^{\prime}\right]=\epsilon \int_{a}^{b}\left(f_{y^{\prime}}-\int_{a}^{x} f_{y} d x\right) \eta^{\prime} d x,
$$

which is linear and continuous of the zeroth order. It is evident that the expression

$$
f_{y}-\frac{d}{d x} f_{y^{\prime}}
$$

is the Volterra derivative of the integral $J$ considered as a function of $y(x)$, and it is approached with the second order.* Similarly

$$
f_{y^{\prime}}-\int_{a}^{x} f_{y} d x
$$

is the derivative of $J$ considered as a function of $y^{\prime}(x)$. It is approached with the zeroth order.

This can easily be extended to functions of surfaces. For convenience it will be supposed that the function $U[z(x, y)]$ is linear and continuous of the second order, and that $z(x, y)$ is of class $C^{(2)}$ and defined over the region $(0 \leqq x \leqq 1$; $0 \leqq y \leqq 1$ ). It follows immediately that

* For a definition of the order of approach see Fischer, Amer. Jour. of Mathematics, vol. 35, no. 4 (1913), p. 383. 


$$
\begin{aligned}
z(x, y) & =\int_{0}^{x} d x_{1} \int_{0}^{y} z_{x y}\left(x_{1}, y_{1}\right) d y_{1}+\int_{0}^{y} d y_{1} \int_{0}^{y_{1}} z_{y y}\left(0, y_{2}\right) d y_{2} \\
+ & \int_{0}^{x} d x_{1} \int_{0}^{x_{1}} z_{x x}\left(x_{2}, 0\right) d x_{2}+z_{y}(0,0) y+z_{x}(0,0) x+z(0,0) .
\end{aligned}
$$

Proceeding as before,

$$
\begin{aligned}
U[z(x, y)]=V\left[z_{x y}(x, y)\right]+V_{1}\left[z_{y y}(0, y)\right]+V_{2}\left[z_{x x}(x, 0)\right] \\
\\
+z_{y}(0,0) U[y]+z_{x}(0,0) U[x]+z(0,0) U[1],
\end{aligned}
$$

where $V, V_{1}$ and $V_{2}$ are linear and continuous of the zeroth order and $U[y], U[x]$ and $U[1]$ are constants.

Columbia University, June, 1916 .

\title{
THE EQUATION OF A PLANE RATIONAL CURVE DEFINED BY PARAMETRIC EQUATIONS.
}

\author{
BY HERBERT WILLIAM RICHMOND.
}

The explicit equation $F(x, y, z)=0$ of a rational plane curve, defined by the parametric equations

$$
x: y: z=A(t): B(t): C(t)
$$

where $A(t), B(t), C(t)$ are polynomials of order $n$ in $t$, is expressed by Salmon (Higher Plane Curves, $\S 44$ ) as a determinant of $3 n$ rows. In the April number of the Bulletin Professor J. E. Rowe exhibits this equation as a symmetrical determinant of $n$ rows, in which each element is a linear function of $x, y, z$. It has been my custom when lecturing upon algebraic geometry to obtain this form of equation as follows:

Let $(x, y, z)$ be the coordinates of the point whose parameter is $t$, and let $s$ be any value of the parameter; then

$$
\left|\begin{array}{lll}
x & A(t) & A(s) \\
z & C(t) & C(s) \\
y & B(t) & B(s)
\end{array}\right|(=\Delta \mathrm{say})
$$

vanishes for every value of $s$. Imagine the determinant expanded and the factor $s-t$ removed. We have now a 